\title{
Scattering of core-shell nanowires with the interference of electric and magnetic resonances
}

\author{
Wei Liu, ${ }^{1,2, *}$ Andrey E. Miroshnichenko, ${ }^{1}$ Rupert F. Oulton, ${ }^{2}$ Dragomir N. Neshev, ${ }^{1}$ Ortwin Hess, ${ }^{2}$ \\ and Yuri S. Kivshar ${ }^{1}$ \\ ${ }^{1}$ Nonlinear Physics Centre and Centre for Ultrahigh-bandwidth Devices for Optical Systems (CUDOS), Research School of \\ Physics and Engineering, Australian National University, Canberra, ACT 0200, Australia \\ ${ }^{2}$ The Blackett Laboratory, Department of Physics, Imperial College London, London SW7 2AZ, United Kingdom \\ *Corresponding author: wli124@physics.anu.edu.au
}

Compiled June 15, 2013

\begin{abstract}
We study the scattering of normally incident waves by core-shell nanowires, which support both electric and magnetic resonances. Within such nanowires, for $p$-polarized incident waves, each electric resonance corresponds to two degernerate scattering channels while the magnetic resonance corresponds to only one channel. Consequently when the electric dipole and magnetic dipole are tuned to overlap spectrally, the magnitude of the electric dipole is twice that of the magnetic one, leading to a pair of angles of vanishing scattering. We further demonstrate that the scattering features of nanowires are polarization dependent, and vanishing scattering angles can also be induced by Fano resonances due to the interference of higher order electric modes with the broad magnetic dipole mode. (C) 2013 Optical Society of America
\end{abstract}

OCIS codes: 290.0290, 240.6680, 260.5740.

Investigations on scattering of light by subwavelength particles are playing a fundamental role in many fields, with applications in optical communications, meteorology, sensing, and other interdisciplinary fields including medical and biological researches [1-3]. For most scattering problems, efficient shaping of the scattering pattern is one of the most crucial issues and is vitally important for various applications, such as nanoantennas $[4,5]$ and photovoltaic devices [6]. However, currently most approaches on the scattering shaping rely on the engineering of electric responses of nanostructures, due to the fact that most materials have only dominant electric responses $[1,2,7,8]$.

It is known that the presence of magnetic resonances in scattering systems brings extra freedom for the scattering engineering due to the interplay of both electric and magnetic responses, of which the Kerker's proposal of serves as a good example [9]. However, in nature only a limited number of materials exhibit magnetic properties at optical frequencies. These are mainly due to magnetic dipole transitions in e.g. rare-earth atoms and are known to only operate in a narrow spectral range, being accompanied by high intrinsic losses.

Recently, inspired by the emerging field of metamaterials, many non-magnetic structures were proven to support artificial magnetic resonances, of which the high permittivity nanowires and spheres are outstanding examples [10-20]. The existence of both electric and magnetic resonances in such high permittivity nanoparticles has led to the demonstration of novel scattering phenomena [18-24], including the vanishing backward scattering proposed by Kerker [7]. However for those demonstrations the electric and magnetic dipoles were mostly spectrally separated and consequently the total scattering was in the non-resonant regime with reduced total mag- nitude. It is not until recently that the vanishing backward scattering in the resonant super-scattering regime was achieved through overlapping an electric dipole (ED) and a magnetic dipole (MD) of the same magnitude in core-shell nanospheres $[25,26]$. Following these first ideas, it is now becoming increasingly important to apply the super-scattering regime to scatterers with other geometries and explore the polarization effects of the scattering as well as the influence of the higher order modes on the scattering pattern.

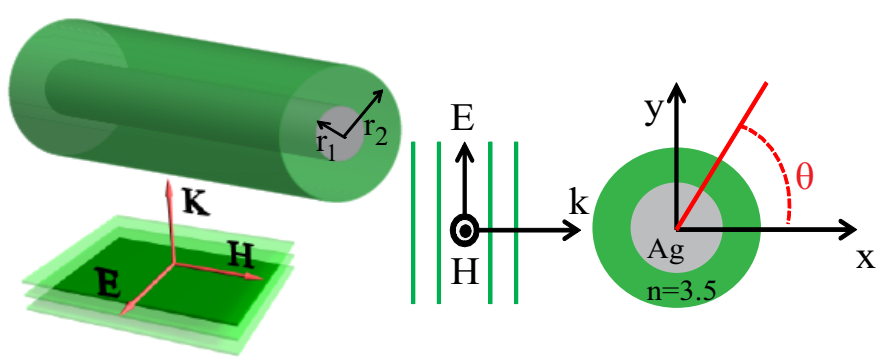

Fig. 1. Schematic of scattering of normally incident $p$ polarized plane waves by a core-shell nanowire. The core nanowire is silver of radius $r_{1}$ and the shell is $n=3.5$ dielectric of radius $r_{2}$. The scattering angle is defined as $\theta$.

In this Letter, we study the scattering of (metal) core(high permittivity dielectric) shell nanowires, which support both electric and magnetic resonances. We find that for $p$-polarized incident waves, the ED and MD can be tuned to overlap spectrally. As the ED corresponds to two degenerate scattering channels while the MD corresponds to only one channel, its magnitude is twice the magnitude of the MD. Consequently, in sharp contrast to spherical structures investigated before, where the scattering is suppressed only at the backward direction, here 

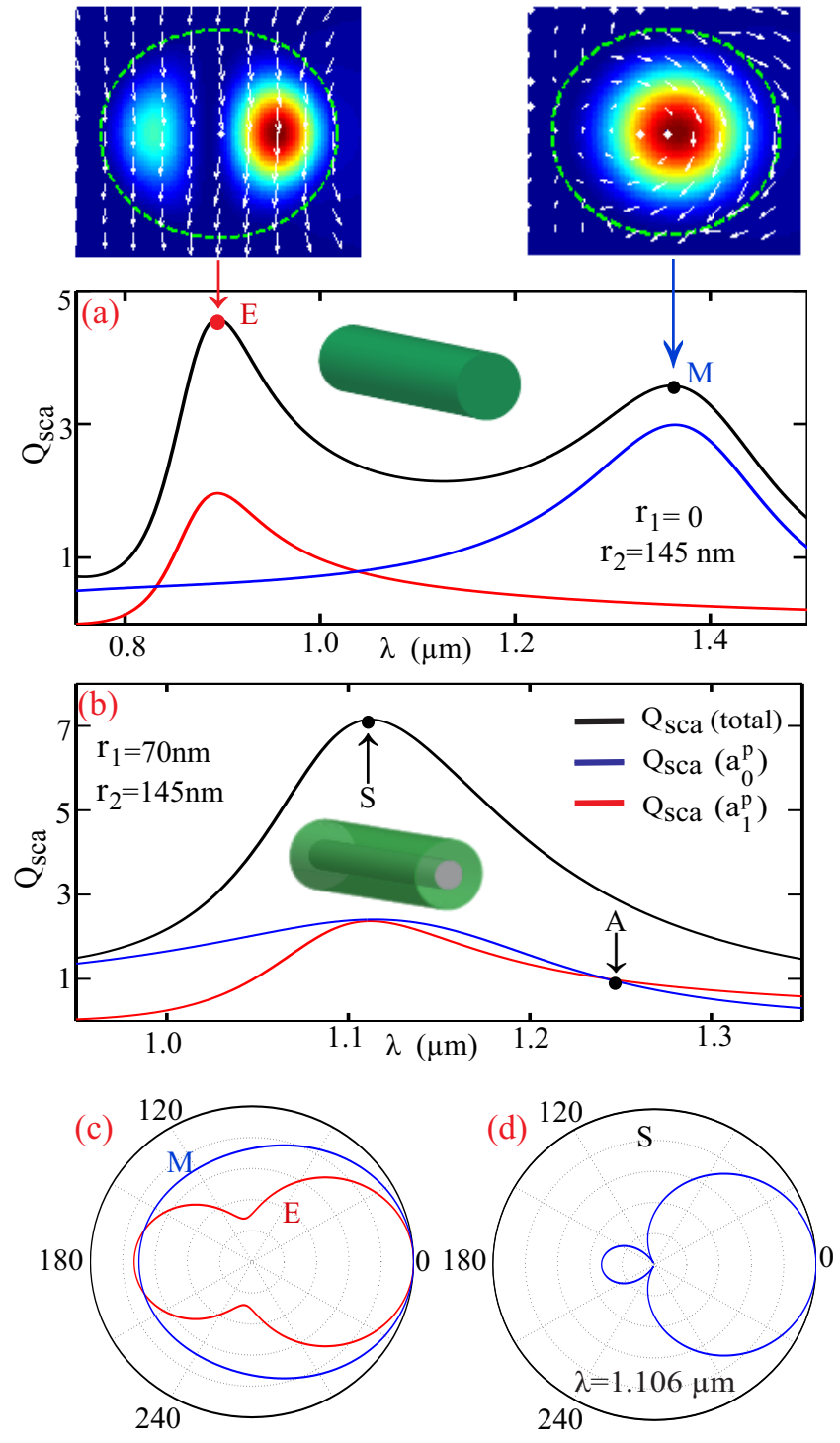

Fig. 2. Scattering efficiency spectra of $p$-wave (total and the contribution from $a_{0}^{p}$ and $a_{1}^{p}$ ) for (a) uniform dielectric nanowire with $r_{1}=0, r_{2}=145 \mathrm{~nm}$ and (b) (silver) core - $(n=3.5$ dielectric $)$ shell nanowire with $r_{1}=70 \mathrm{~nm}$ and $r_{2}=145 \mathrm{~nm}$. Above (a) the near-field distributions of $\left|H_{z}\right|^{2}$ (colormap) and transverse electric field $E_{t}$ (arrows) at points $\mathrm{E}$ and $\mathrm{M}$ are shown. (c) and (d) show the scattering patterns for the points indicated in (a) and (b): $\lambda_{S}=1.106 \mu \mathrm{m}$, $\lambda_{A}=1.242 \mu \mathrm{m}, \lambda_{E}=0.895 \mu \mathrm{m}$, and $\lambda_{M}=1.363 \mu \mathrm{m}$.

we demonstrate a pair of angles along which the scattering is vanishing. It is also demonstrated that the scattering features are highly polarization dependent, and the vanishing scattering angles can also be obtained through the Fano resonance, which is induced by the interference of the broad MD and the narrow higher order electric modes.

The structure we study is shown schematically in Fig. 1. The infinitely long nanowire is arranged along $z$ direction with a silver core (the permittivity is from Ref. [27]) and a dielectric shell $(n=3.5)$. Here the incident plane wave propagates along $x$ direction and the scattered light is confined at the $x-y$ plane. The electric field of the incident wave is either polarized along $y(p$ wave) or along $z$ ( $s$-wave). The scattering of a nanowire (single-layered or multi-layered) can be solved analytically and the scattering efficiency is $[1,2]$ :

$$
Q_{s c a}^{s, p}=\frac{2}{k r}\left[\left(a_{0}^{s, p}\right)^{2}+2 \sum_{m=1}^{\infty}\left(a_{m}^{s, p}\right)^{2}\right],
$$

where $k$ is the angular wave-number in the background material (vacuum in this study); $r$ is the radius of the outmost layer; $a_{0}$ and $a_{m}$ are the scattering coefficients, and the superscripts $s$ and $p$ corresponds to $s$-wave and $p$-wave, respectively. More specifically $a_{0}$ and $a_{1}$ correspond to the magnetic (electric) and electric (magnetic) dipoles for $p(s)$ waves, respectively [11]. The scattering coefficient $a_{m}$ relates to an angular field distribution of $e^{\operatorname{im} \theta}$ [11], which corresponds to two angular momentum channels with angular momentums $\pm m \hbar$, where the scattering angle $\theta$ is defined in Fig. 1. In contrast $a_{0}$ corresponds to a uniform angular field distribution with no angular momentum along $z$ direction $[1,2]$. As a result, the contribution from $a_{m}$ to the scattering efficiency is twice that from $a_{0}$. At the same time the angular scattering amplitude can be expressed as $[1,2]$ :

$$
S A^{s, p}(\theta)=\sqrt{2 / \pi k}\left|a_{0}^{s, p}+2 \sum_{m=1}^{\infty} a_{m}^{s, p} \cos (m \theta)\right| .
$$

In Fig. 2 we show the scattering efficiency spectra (SES) for incident $p$-wave, including the total scattering efficiency and the contributions from $a_{0}^{p}$ (MD) and $a_{1}^{p}$ (single channel, ED). Firstly we study a single-layered nanowire $\left(r_{1}=0, r_{2}=145 \mathrm{~nm}\right)$ and the SES is shown in Fig. 2(a). It is clear that although both dominant ED and MD are supported with resonances centered at point $\mathrm{E}$ and $\mathrm{M}$, respectively, they are spectrally separated. Above Fig. 2(a) we also show the near-field distributions of longitudinal magnetic field intensity $\left(\left|H_{z}\right|^{2}\right.$,colormap) and transverse electric field ( $E_{t}$, arrows) of points $\mathrm{E}$ and $\mathrm{M}$. It is clear that ED and MD are supported at points $\mathrm{E}$ and $\mathrm{M}$ respectively: at point $\mathrm{E}$, the $H_{z}$ field shows a typical dipolar distribution with transverse electric filed almost linearly polarized; at point $\mathrm{M}, H_{z}$ field is almost azimuthally symmetric inside the nanowire, accompanied by circulating displacement currents, indicating the existence of an artificial magnetic dipole [12-16]. Fig. 2(c) shows the scattering patterns at both points. Typical dipole-like scattering patterns are observed: at point $\mathrm{E}$ the ED is dominant and oriented along $y$ direction with a two-lobe scattering pattern in the $x-y$ plane; at point $\mathrm{M}$ the MD is dominant and oriented along $z$ direction with an almost circular scattering pattern in the $x-y$ plane. Then we study a two-layered core-shell nanowire, shown in Fig. 1. As shown in Fig. 2(b) it is clear that when the inner radius $r_{1}=70 \mathrm{~nm}$ and the outer radius $r_{2}=145 \mathrm{~nm}$, the ED and MD can overlap and thus creating a superscattering spectral regime (the corresponding single channel limit in terms of scattering efficiency is approximately 2.4) [28]. We note here that the overlapping wavelength of $\mathrm{ED}$ and $\mathrm{MD}$ for such structures is 

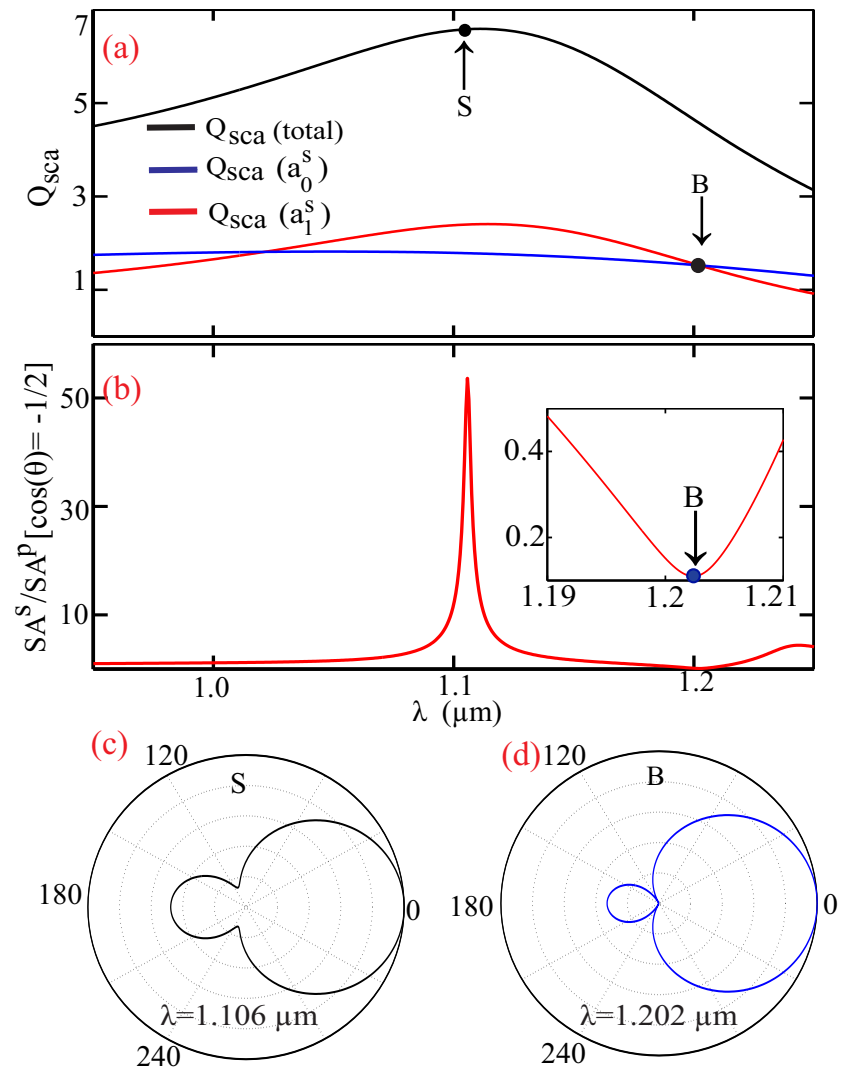

Fig. 3. (a) Same as in Fig. 2(b) but for incident $s$-wave. (b) The ratio of $S A^{s} / S A^{p}$ with equal $p$-wave and $s$-wave incidence along $\theta=120^{\circ}$ and $240^{\circ}$. The inset shows an enlarged area of $\lambda=1.190-1.210 \mu \mathrm{m}$. (c) and (d) show the scattering patterns for the points indicated in (a) and (b) with $\lambda_{B}=1.202 \mu \mathrm{m}$ and $\lambda_{S}=1.106 \mu \mathrm{m}$.

highly tunable, and can be shifted to shorter or longer wavelengths for smaller or larger scales of the nanowires with specific aspect ratios. Also in the spectral regime in Fig. 2(b), the ED and MD are dominant and Eq. (2) can be simplified as:

$$
S A^{p}(\theta)=\sqrt{2 / \pi k}\left|a_{0}^{p}+2 a_{1}^{p} \cos (\theta)\right| .
$$

At the overlapping resonant point $\mathrm{S}$, as indicated in Fig. 2(b), both $a_{0}^{p}$ and $a_{1}^{p}$ are real [1] and $a_{0}^{p}=a_{1}^{p}=b$. Thus at this point we have $S A^{p}(\theta)=b \sqrt{2 / \pi k} \mid 1+$ $2 \cos (\theta) \mid$, which indicates that when $\theta=120^{\circ}, 240^{\circ}$ $[\cos (\theta)=-1 / 2]$ the scattering is vanishing. In Fig. $2(\mathrm{~d})$ we show the normalized scattering amplitude at point S. We note that at point A indicated in Fig. 2(b), the scattering pattern is identical as that of point $\mathrm{S}$. The difference is that point $\mathrm{A}$ is in the non-resonant regime and the overall scattering is less than half of that at point S.

Next we change the incident wave to $s$-wave and show the SES for the core-shell nanostructure in Fig. 3(a). In contrast to $p$-wave incidence, at the point $\mathrm{S},\left|a_{0}^{p}\right| \neq\left|a_{1}^{p}\right|$. Thus the scattering at the vanishing scattering angles of the $p$-wave can not be neglected, as demonstrated in Fig. 3(c). This feature is similar to the reflection at the Brewster angle, which is highly polarization de-
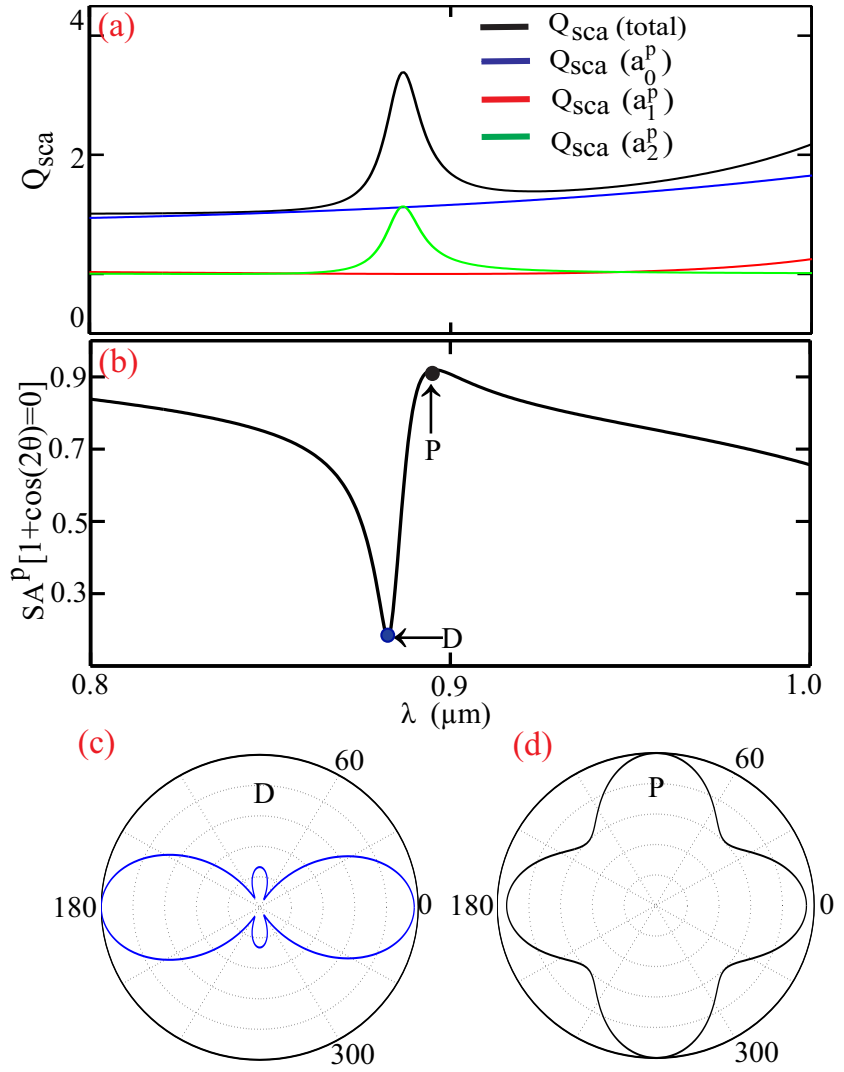

Fig. 4. (a) Same as in Fig. 2(b) but in the spectral regime of $\lambda=0.8-1 \mu \mathrm{m}$. (b) normalized scattering amplitude along $\theta=60^{\circ}, 120^{\circ}, 240^{\circ}$ and $300^{\circ}$. (c) and (d) show the scattering patterns for the points indicated in (b) with $\lambda_{D}=0.883 \mu \mathrm{m}$ and $\lambda_{P}=0.895 \mu \mathrm{m}$.

pendent. In Fig. 3(b) we show the amplitude ratio of $S A^{s} / S A^{p}[\cos (\theta)=-1 / 2]$ with equal $p$-wave and $s$-wave incidence. There is a sharp peak centered at point $S$ where the $p$-wave scattering is negligible [Fig. $2(\mathrm{~d})$ ] compared to that of the $s$-wave incidence along $\theta=120^{\circ}$ and $240^{\circ}$ (the maximum intensity ratio can reach approximately 3500$)$. At the same time, the width of such response is as narrow as $5 \mathrm{~nm}$, indicating that it might be used for efficient polarization splitter and filters. We also note that there is a dip located at point $B$ [inset of Fig. 3(b)], where the scattering of $s$-wave incidence along $\theta=120^{\circ}$ and $240^{\circ}$ is vanishing [Fig. 3(d)].

Finally, we study the interference of MD and the electric quadruple mode (QM, characterized by $a_{2}$ ) for $p$ wave. In Fig. 4(a) we show the SES for the same coreshell structure at the spectral range of 750-1050 nm. The contribution from QM and MD are dominant and then Eq. (2) can be expressed as:

$$
S A^{p}(\theta)=\sqrt{2 / \pi k}\left|a_{0}^{p}+2 a_{2}^{p} \cos (2 \theta)\right| .
$$

Compared to the QM, the MD response is rather broad, and its interference with the narrow QM will produce sharp Fano resonances $[8,29,30]$. In Fig. 4(b) we show the normalized scattering amplitude when $1+\cos (2 \theta)=0$, and the two modes can almost cancel each other, re- 
sulting in vanishing scattering along two pairs of angles: $\theta=60^{\circ}, 120^{\circ}, 240^{\circ}$ and $300^{\circ}$. Typical Fano asymmetric line-shape is observed. The specific scattering patters for the Fano dip (point D) and Fano peak (point P) are shown in Fig. 4(c) and Fig. 4(d), respectively, showing clearly the destructive and constructive interferences along those angles.

In summary, we have studied scattering of core-shell nanowires, which support both electric and magnetic resonances. We have demonstrated how to achieve a pair of vanishing scattering angles in the superscattering regime of overlapped ED and MD for $p$-waves. We have also demonstrated the polarization dependence of these scattering features and Fano resonance induced vanishing scattering angles. Our study generalizes the proposal of Kerker for backward scattering suppression, and our approaches can be extended to higher order modes or to other non-cylindrical structures where differnt vanishing scattering angles can be obtained in the resonant strong-scattering regimes. Our results shed new light on the direction of scattering shaping based on interferences of both electric and magnetic resonances, which can play a major role in applications such as nanoantennas, nanolasers, sensors, solar cells and so on.

We acknowledge a support from the Australian Research Council and the Leverhulme Trust.

\section{References}

1. M. Kerker, The scattering of light, and other electromagnetic radiation (Academic Press, New York, 1969).

2. C. F. Bohren and D. R. Huffman, Absorption and scattering of light by small particles (Wiley, New York, 1983).

3. R. Huschka, J. Zuloaga, M. W. Knight, L. V. Brown, P. Nordlander, and N. J. Halas, J. Am. Chem. Soc. 133, 12247 (2011).

4. L. Novotny and N. van Hulst, Nat. Photon. 5, 83 (2011).

5. A. G. Curto, G. Volpe, T. H. Taminiau, M. P. Kreuzer, R. Quidant, and N. F. van Hulst, Science 329, 930 (2010).

6. H. A. Atwater and A. Polman, Nat. Mater. 9, 865 (2010).

7. B. S. Luk'yanchuk and V. Ternovsky, Phys. Rev. B 73, 235432 (2006).

8. B. S. Luk'yanchuk, A. E. Miroshnichenko, and Y. S. Kivshar, J. Opt. 15, 073001 (2013).

9. M. Kerker, D. S. Wang, and C. L. Giles, J. Opt. Soc. Am. 73, 765 (1983).

10. L. Peng, L. Ran, H. Chen, H. Zhang, J. A. Kong, and T. M. Grzegorczyk, Phys. Rev. Lett. 98, 157403 (2007).

11. K. Vynck, D. Felbacq, E. Centeno, A. I. Cabuz, D. Cassagne, and B. Guizal, Phys. Rev. Lett. 102, 133901 (2009).

12. A. B. Evlyukhin, C. Reinhardt, A. Seidel, B. S. Luk'yanchuk, and B. N. Chichkov, Phys. Rev. B 82, 045404 (2010).

13. A. Garcia-Etxarri, R. Gomez-Medina, L. S. FroufePerez, C. Lopez, L. Chantada, F. Scheffold, J. Aizpurua, M. Nieto-Vesperinas, and J. J. Saenz, Opt. Express 19, 4815 (2011).

14. R. Gomez-Medina, B. Garcia-Camara, I. Suarez-Lacalle,
F. Gonzalez, F. Moreno, M. Nieto-Vesperinas, and J. J. Saenz, J. Nanophotonics 5, 053512 (2011).

15. A. I. Kuznetsov, A. E. Miroshnichenko, Y. H. Fu, J. B. Zhang, and B. S. Lukyanchuk, Sci. Rep. 2, 492 (2012).

16. A. B. Evlyukhin, S. M. Novikov, U. Zywietz, R. L. Eriksen, C. Reinhardt, S. I. Bozhevolnyi, and B. N. Chichkov, Nano Lett. 12, 3749 (2012).

17. R. Paniagua-Dominguez, F. Lopez-Tejeira, R. Marques, and J. A. Sanchez-Gil, New. J. Phys. 13, 123017 (2011).

18. J. A. Schuller, R. Zia, T. Taubner, and M. L. Brongersma, Phys. Rev. Lett. 99, 107401 (2007).

19. P. Y. Fan, U. K. Chettiar, L. Y. Cao, F. Afshinmanesh, N. Engheta, and M. L. Brongersma, Nat. Photon. 6, 380 (2012).

20. R. Paniagua-Dominguez, D. R. Abujetas, and J. A. Sanchez-Gil, Sci. Rep. 3, 1057 (2013).

21. D. S. Filonov, A. E. Krasnok, A. P. Slobozhanyuk, P. V. Kapitanova, E. A. Nenasheva, Y. S. Kivshar, and P. A. Belov, Appl. Phys. Lett. 100, 201113 (2012).

22. J. M. Geffrin, B. Garcia-Camara, R. Gomez-Medina, P. Albella, L. S. Froufe-Perez, C. Eyraud, A. Litman, R. Vaillon, F. Gonzalez, M. Nieto-Vesperinas, J. J. Saenz, and F. Moreno, Nat. Commun. 3, 1171 (2012).

23. Y. H. Fu, A. I. Kuznetsov, A. E. Miroshnichenko, Y. F. Yu, and B. Lukyanchuk, Nat. Commun. 4, 1527 (2013).

24. S. Person, M. Jain, Z. Lapin, J. J. Senz, G. Wicks, and L. Novotny, Nano Lett. 13, 1806 (2013).

25. W. Liu, A. E. Miroshnichenko, D. N. Neshev, and Y. S. Kivshar, ACS Nano 6, 5489 (2012).

26. W. Liu, A. E. Miroshnichenko, D. N. Neshev, and Y. S. Kivshar, Phys. Rev. B 86, 081407(R) (2012).

27. P. B. Johnson and R. W. Christy, Phys. Rev. B 6, 4370 (1972).

28. Z. C. Ruan and S. H. Fan, Phys. Rev. Lett. 105, 013901 (2010).

29. A. E. Miroshnichenko, S. Flach, and Y. S. Kivshar, Rev. Mod. Phys. 82, 2257 (2010).

30. B. Luk'yanchuk, N. I. Zheludev, S. A. Maier, N. J. Halas, P. Nordlander, H. Giessen, and C. T. Chong, Nat. Mater. 9, 707 (2010). 


\section{References}

1. M. Kerker, The scattering of light, and other electromagnetic radiation (Academic Press, New York, 1969).

2. C. F. Bohren and D. R. Huffman, Absorption and scattering of light by small particles (Wiley, New York, 1983).

3. R. Huschka, J. Zuloaga, M. W. Knight, L. V. Brown, P. Nordlander, and N. J. Halas, "Light-induced release of dna from gold nanoparticles: Nanoshells and nanorods," J. Am. Chem. Soc. 133, 12247 (2011).

4. L. Novotny and N. van Hulst, "Antennas for light," Nat. Photon. 5, 83 (2011).

5. A. G. Curto, G. Volpe, T. H. Taminiau, M. P. Kreuzer, R. Quidant, and N. F. van Hulst, "Unidirectional emission of a quantum dot coupled to a nanoantenna," Science 329, 930 (2010).

6. H. A. Atwater and A. Polman, "Plasmonics for improved photovoltaic devices," Nat. Mater. 9, 865 (2010).

7. B. S. Luk'yanchuk and V. Ternovsky, "Light scattering by a thin wire with a surface-plasmon resonance: Bifurcations of the poynting vector field," Phys. Rev. B 73, $235432(2006)$.

8. B. S. Luk'yanchuk, A. E. Miroshnichenko, and Y. S. Kivshar, "Fano resonances and topological optics: an interplay of far- and near-field interference phenomena," J. Opt. 15, 073001 (2013).

9. M. Kerker, D. S. Wang, and C. L. Giles, "Electromagnetic scattering by magnetic spheres," J. Opt. Soc. Am. 73, 765 (1983).

10. L. Peng, L. Ran, H. Chen, H. Zhang, J. A. Kong, and T. M. Grzegorczyk, "Experimental observation of lefthanded behavior in an array of standard dielectric resonators," Phys. Rev. Lett. 98, 157403 (2007).

11. K. Vynck, D. Felbacq, E. Centeno, A. I. Cabuz, D. Cassagne, and B. Guizal, "All-dielectric rod-type metamaterials at optical frequencies," Phys. Rev. Lett. 102, 133901 (2009).

12. A. B. Evlyukhin, C. Reinhardt, A. Seidel, B. S. Luk'yanchuk, and B. N. Chichkov, "Optical response features of si-nanoparticle arrays," Phys. Rev. B 82, 045404 (2010).

13. A. Garcia-Etxarri, R. Gomez-Medina, L. S. FroufePerez, C. Lopez, L. Chantada, F. Scheffold, J. Aizpurua, M. Nieto-Vesperinas, and J. J. Saenz, "Strong magnetic response of submicron silicon particles in the infrared," Opt. Express 19, 4815 (2011).

14. R. Gomez-Medina, B. Garcia-Camara, I. Suarez-Lacalle, F. Gonzalez, F. Moreno, M. Nieto-Vesperinas, and J. J. Saenz, "Electric and magnetic dipolar response of germanium nanospheres: interference effects, scattering anisotropy, and optical forces," J. Nanophotonics 5 , $053512(2011)$

15. A. I. Kuznetsov, A. E. Miroshnichenko, Y. H. Fu, J. B. Zhang, and B. S. Lukyanchuk, "Magnetic light," Sci. Rep. 2, 492 (2012).

16. A. B. Evlyukhin, S. M. Novikov, U. Zywietz, R. L. Eriksen, C. Reinhardt, S. I. Bozhevolnyi, and B. N. Chichkov, "Demonstration of magnetic dipole resonances of dielectric nanospheres in the visible region," Nano Lett. 12, 3749 (2012).

17. R. Paniagua-Dominguez, F. Lopez-Tejeira, R. Marques, and J. A. Sanchez-Gil, "Metallo-dielectric coreshell nanospheres as building blocks for optical three- dimensional isotropic negative-index metamaterials," New. J. Phys. 13, 123017 (2011).

18. J. A. Schuller, R. Zia, T. Taubner, and M. L. Brongersma, "Dielectric metamaterials based on electric and magnetic resonances of silicon carbide particles," Phys. Rev. Lett. 99, 107401 (2007).

19. P. Y. Fan, U. K. Chettiar, L. Y. Cao, F. Afshinmanesh, N. Engheta, and M. L. Brongersma, "An invisible metal-semiconductor photodetector," Nat. Photon. 6, 380 (2012).

20. R. Paniagua-Dominguez, D. R. Abujetas, and J. A. Sanchez-Gil, "Ultra low-loss, isotropic optical negative-index metamaterial based on hybrid metal-semiconductor nanowires," Sci. Rep. 3, 1057 (2013).

21. D. S. Filonov, A. E. Krasnok, A. P. Slobozhanyuk, P. V. Kapitanova, E. A. Nenasheva, Y. S. Kivshar, and P. A. Belov, "Experimental verification of the concept of alldielectric nanoantennas," Appl. Phys. Lett. 100, 201113 (2012).

22. J. M. Geffrin, B. Garcia-Camara, R. Gomez-Medina, P. Albella, L. S. Froufe-Perez, C. Eyraud, A. Litman, R. Vaillon, F. Gonzalez, M. Nieto-Vesperinas, J. J. Saenz, and F. Moreno, "Magnetic and electric coherence in forward- and back-scattered electromagnetic waves by a single dielectric subwavelength sphere," Nat. Commun. 3, 1171 (2012).

23. Y. H. Fu, A. I. Kuznetsov, A. E. Miroshnichenko, Y. F. $\mathrm{Yu}$, and B. Lukyanchuk, "Directional visible light scattering by silicon nanoparticles," Nat. Commun. 4, 1527 (2013).

24. S. Person, M. Jain, Z. Lapin, J. J. Senz, G. Wicks, and L. Novotny, "Demonstration of zero optical backscattering from single nanoparticles," Nano Lett. 13, 1806 (2013).

25. W. Liu, A. E. Miroshnichenko, D. N. Neshev, and Y. S. Kivshar, "Broadband unidirectional scattering by magneto-electric core-shell nanoparticles," ACS Nano 6, 5489 (2012).

26. W. Liu, A. E. Miroshnichenko, D. N. Neshev, and Y. S. Kivshar, "Polarization-independent fano resonances in arrays of core-shell nanoparticles," Phys. Rev. B 86, 081407(R) (2012).

27. P. B. Johnson and R. W. Christy, "Optical constants of the noble metals," Phys. Rev. B 6, 4370 (1972).

28. Z. C. Ruan and S. H. Fan, "Superscattering of light from subwavelength nanostructures," Phys. Rev. Lett. 105, 013901 (2010).

29. A. E. Miroshnichenko, S. Flach, and Y. S. Kivshar, "Fano resonances in nanoscale structures," Rev. Mod. Phys. 82, 2257 (2010).

30. B. Luk'yanchuk, N. I. Zheludev, S. A. Maier, N. J. Halas, P. Nordlander, H. Giessen, and C. T. Chong, "The fano resonance in plasmonic nanostructures and metamaterials," Nat. Mater. 9, 707 (2010). 